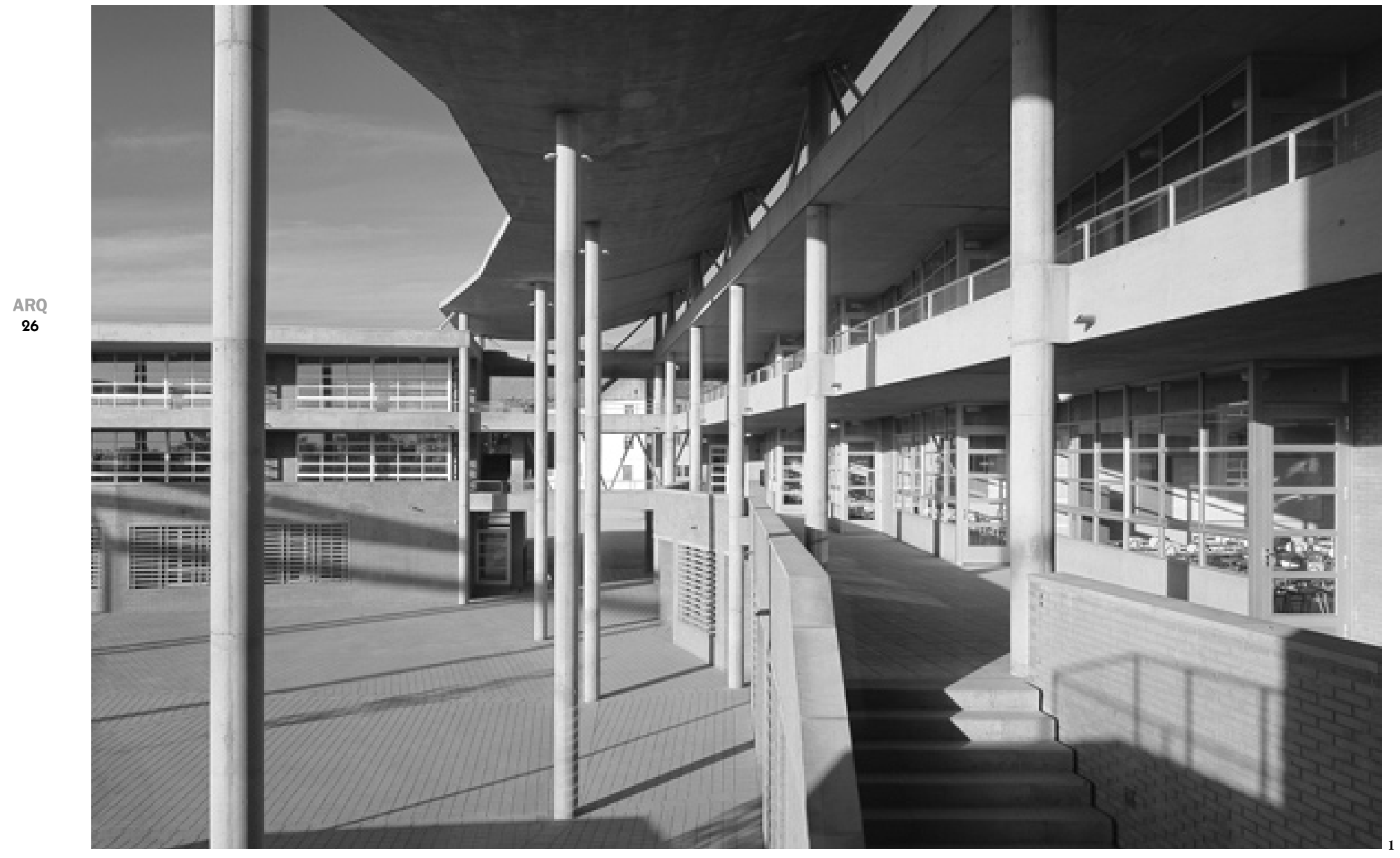

Undurraga \& Devés arquitectos Puente Alto, Chile

Los edificios presentados forman parte del plan de crecimiento de esta institución de beneficencia ligada al Ministerio de Educación chileno. Son dos escuelas que por su emplazamiento generan nuevos centros y lugares de reunión dentro del gran predio que las aloja; proponen otras orientaciones para el conjunto de pabellones existente en el lugar e inauguran nuevas relaciones con los espacios abiertos $y$ los materiales del conjunto.

Palabras clave: Arquitectura - Chile, establecimientos educacionales, colegios, escuelas públicas, internado escolar, Reforma Educacional.

The buildings shown form part of the expansion plan of a charity institution linked to Chile's Ministry of Education. The location of the two schools means they create new center and meeting places within their extensive site, as they re-orient the group of pre-existing buildings, and generate new relationships with the open spaces and the materials of the whole.

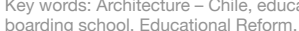

\title{
Escuelas Básicas
}

El principal centro de la Sociedad Protectora de la Infancia se encuentra al sur de la ciudad de Santiago, en la comuna de Puente Alto. Allí, en un territorio de 18 ha, que conserva todavía un cierto aire rural, se construyó en el primer tercio del siglo XX un conjunto de edificios emblemáticos que dan cuenta del período de transición entre la arquitectura neocolonial y los primeros signos de modernidad. En ellos funcionan hoy las distintas dependencias que esta institución mantiene, cuyo objetivo es dar abrigo, educación y dignidad a los niños más pobres del país.

Las dos escuelas que aquí mostramos forman parte de un proyecto mayor de edificación, que busca responder a las necesidades de crecimiento y modernización en que está empeñada la institución. Como plan general se propone el ordenamiento de lo disperso, articulando espacios de encuentro y circulación a partir de la construcción de nuevos edificios que, en su aproximación a los edificios ya existentes, dan origen a plazuelas y calles que evocan episodios urbanos. De este modo el espacio público se constituye en el articulador de los distintos períodos de la historia.

Los nuevos edificios se han dispuesto de tal forma de conformar un espacio público de encuentro -una plaza-, que nos refiere al espacio urbano entendido en un sentido histórico.

A la economía formal de las fachadas públicas de las nuevas escuelas se sumó una restricción en la altura de modo que los volúmenes propuestos no sobrepasaran los ya existentes. $\mathrm{Al}$ interior del nuevo conjunto se propuso una geometría más azarosa, creando un paisaje lúdico de hormigón y ladrillo, propicio para aprender y jugar, en contraste con la síntesis formal de las fachadas externas, caracterizadas por un zócalo de ladrillo y una celosía prefabricada de hormigón.

Se ha considerado el hormigón a la vista como el material protagónico con que se materializaron estas escuelas. Para nosotros el hormigón representa la atemporalidad propia de la piedra. Con esto no queremos desconocer la libertad e infinitas posibilidades formales propias de la plasticidad del material. Sin embargo, es la posibilidad de trascender al tiempo, el arraigo al suelo junto a la posibilidad de expresar de manera simultánea el equilibrio entre la estructura del edificio como soporte y el acabado de fachada, lo que motiva el uso del hormigón visto en buena parte de nuestros últimos trabajos. El hormigón como ningún otro material nos provee la posibilidad de conjugar en un instante preciso lo primitivo y lo tecnológico. De allí deriva buena parte de su poética. ARQ 


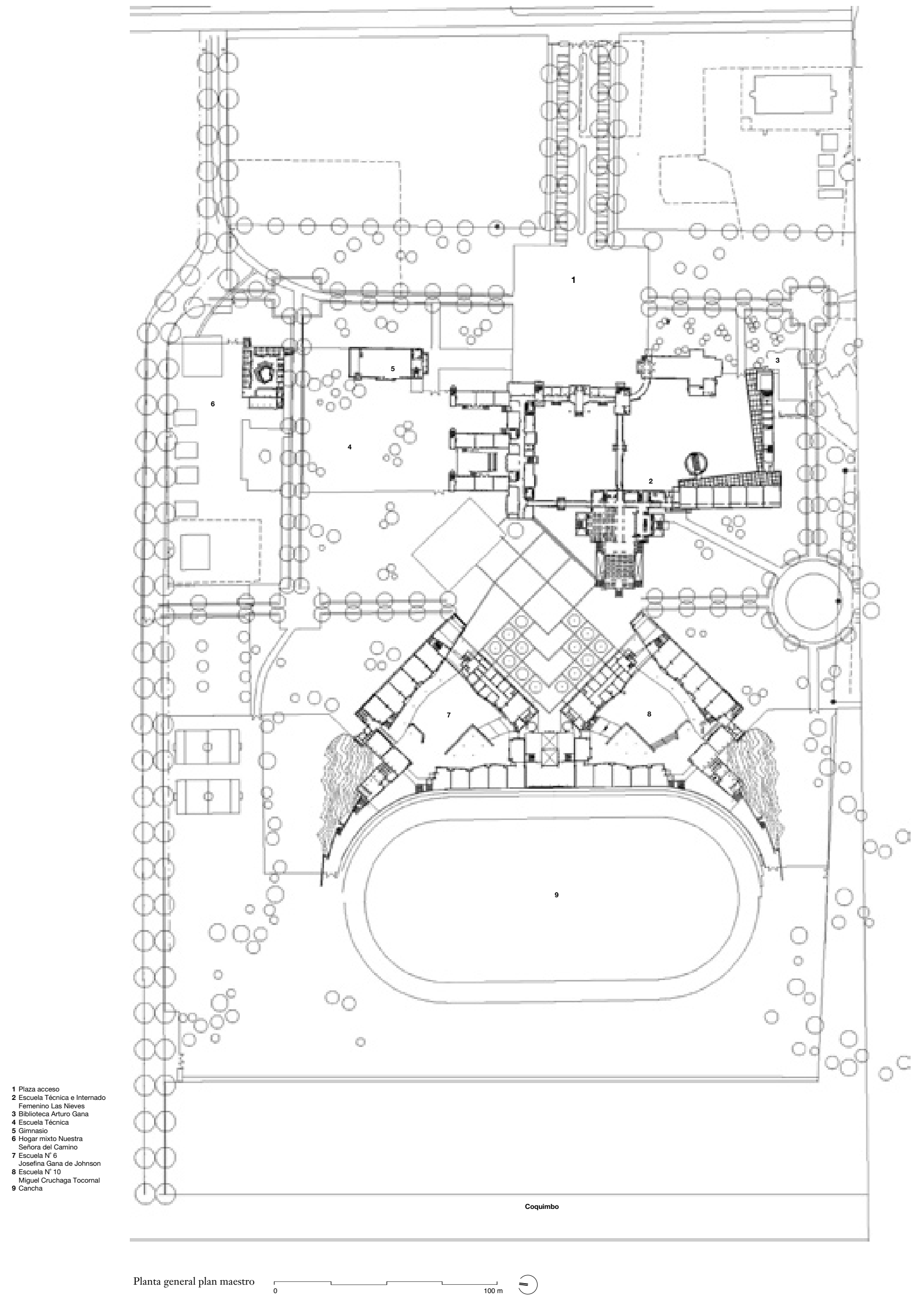



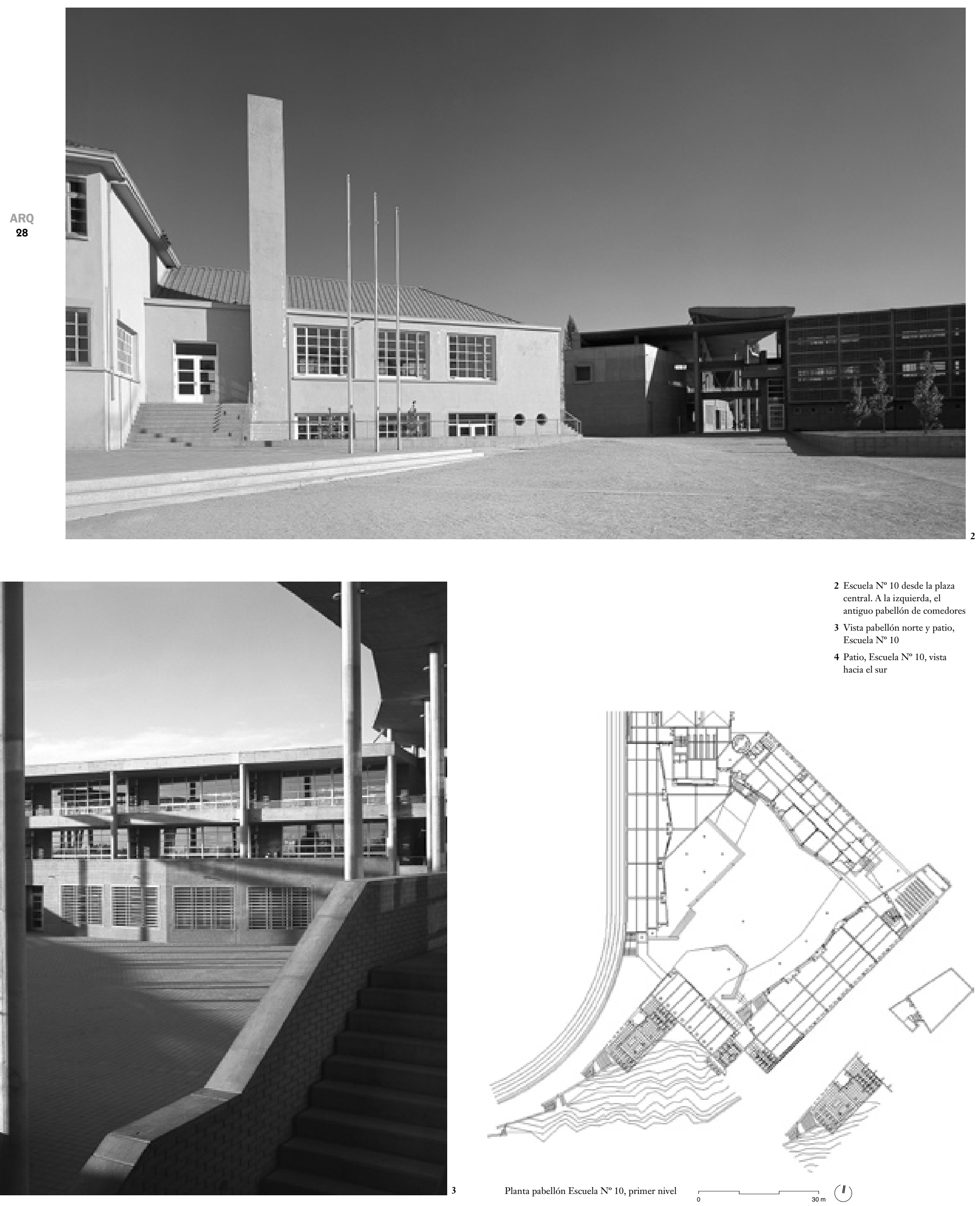

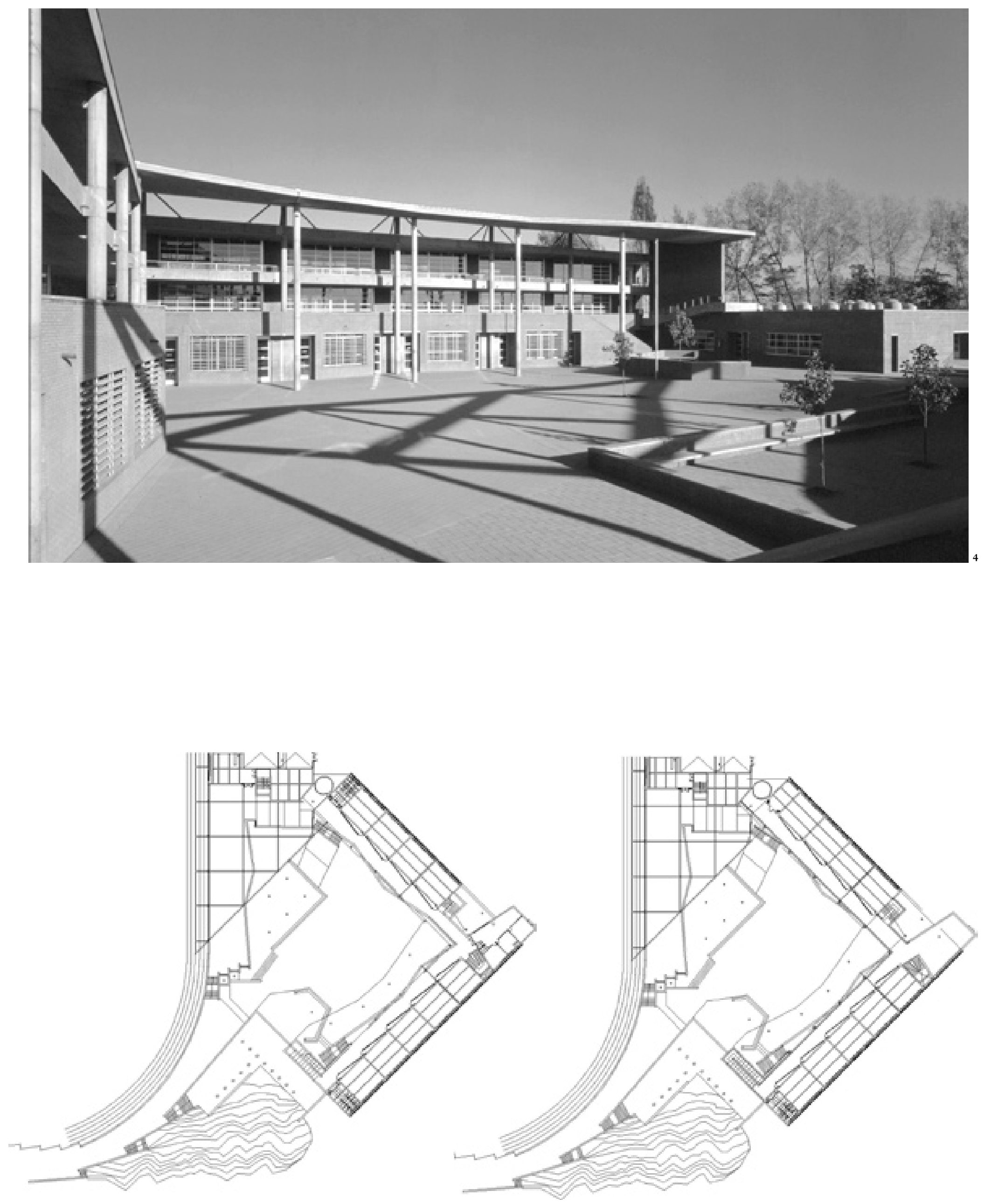


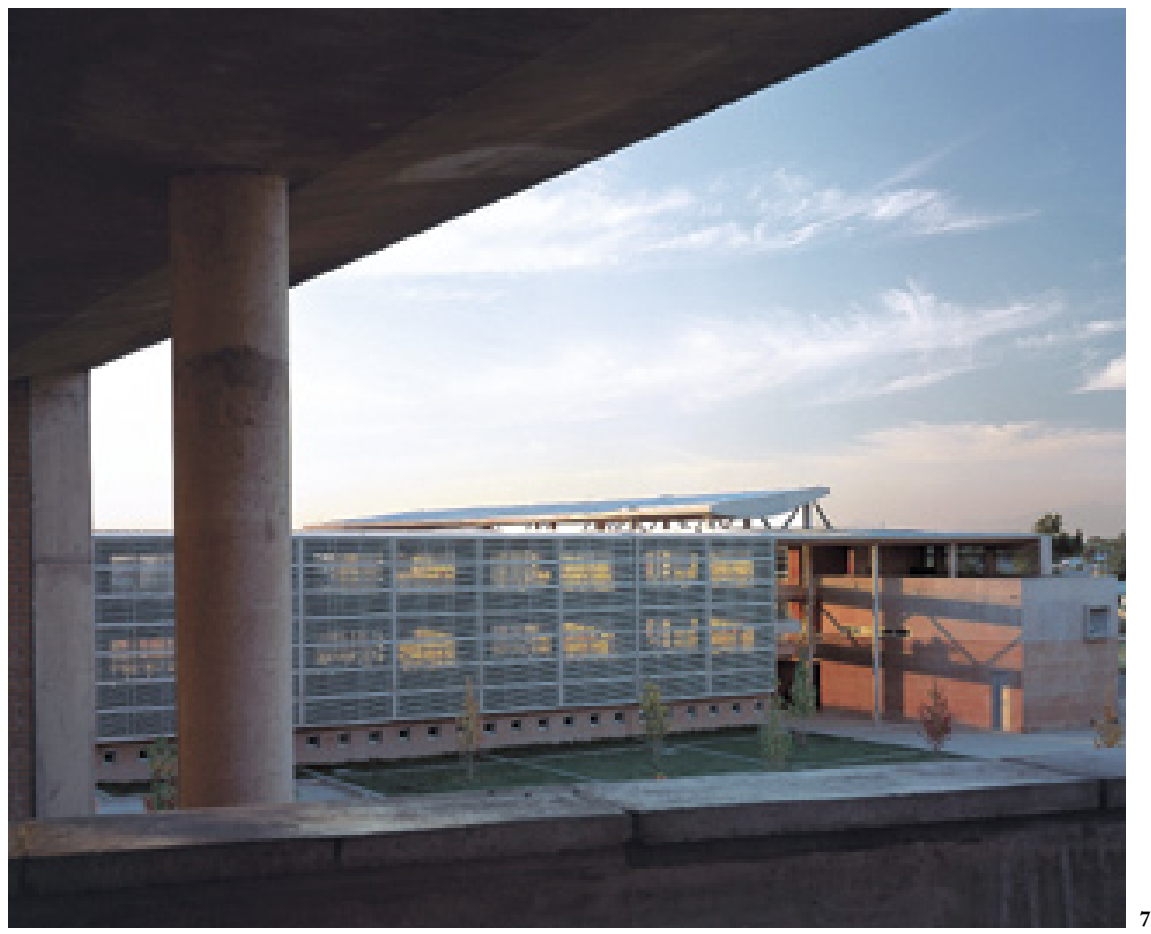

\section{Escuelas Básicas}

Arquitectos Undurraga \& Devés arquitectos Alicia Ross, Cristián Undurraga y Ana Luisa Devés Colaboradores Rodrigo Rojas y Felipe Dufeu Ubicación Av. Concha y Toro 1898 / 2140, Puente Alto, Santiago, Chile

Cliente Sociedad Protectora de la Infancia

Cálculo estructural José Jiménez y Rafael Gatica Construcción Mena y Ovalle

Materialidad Hormigón armado, revestimiento de ladrillo, celosías prefabricadas de hormicón armado Presupuesto de la obra 13 UF/ m² (US\$316/ m²)

Superficie terreno 18 ha

Superficie construida $10.000 \mathrm{~m}^{2}$

Año proyecto 2000

Año construcción 2001

7 Fotografía Guy Wenborne
5 Acceso desde plaza,

Escuela $\mathrm{N}^{\circ} 10$

6 Corredor en segundo nivel,

bloque de aulas

7 Vista desde Escuela $\mathrm{N}^{\circ} 10$ a Escuela $\mathrm{N}^{\circ} 5$, a través de la plaza

8 Fachada norte, Escuela $\mathrm{N}^{\circ} 5$ Celosías de hormigón prefabricadas

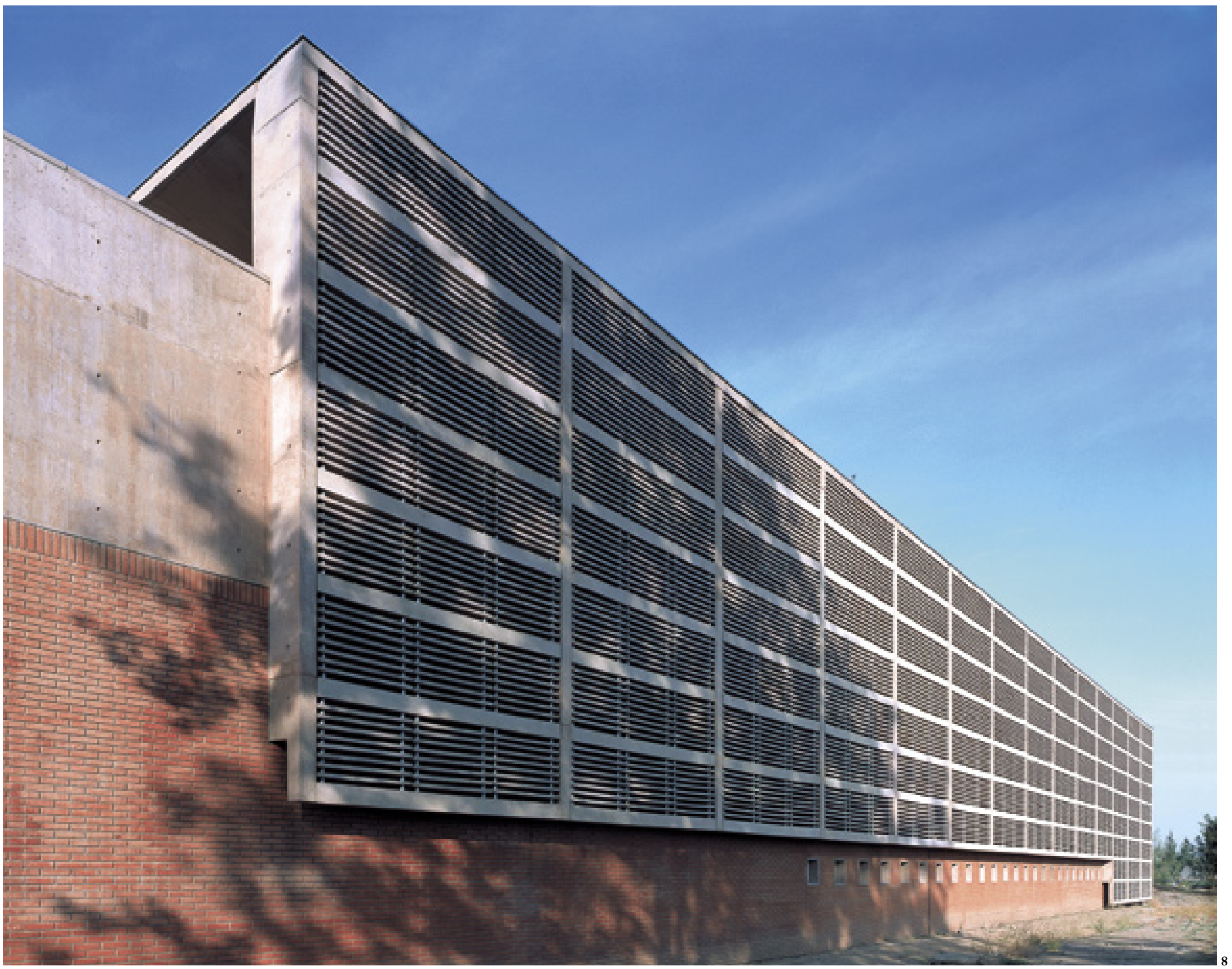

